\title{
Adoption of Digital Pathology in Developing Countries: From Benefits to Challenges
}

\author{
Talat Zehra and Asma Shabbir \\ Department of Pathology, Jinnah Sindh Medical University, Karachi, Pakistan
}

\begin{abstract}
Digital pathology and the use of artificial intelligence constitute undisputedly the future of modern pathology. The outcomes and benefits of the whole slide imaging are beyond the scope of traditional microscopy, which the pathologists were using for decades. COVID-19 pandemic has further highlighted the importance of digital pathology as it offers the pathologists to work from their place of comfort and bridges the gap of physical barriers. In addition to the many advantages, there are certain limitations and challenges, which have to be overcomed particularly in the developing world. The major issue is the cost of scanners and technical support and training of staff. However, despite all these problems and challenges that exist, these can be resolved with the passage of time, where the role of world leader organisations will be of great importance in resolving these challenges.
\end{abstract}

Key Words: Digital pathology, Artificial intelligence, Whole slide imaging.

How to cite this article: Zehra T, Shabbir A. Adoption of Digital Pathology in Developing Countries: From Benefits to Challenges. J Coll Physicians Surg Pak 2021; 31(09):1120-1122.

\section{INTRODUCTION}

Virtual images or digital slides are nowadays widely used by pathologists all over the world for knowledge sharing, consultation and research purpose by using microscope connected cameras and also from mobile cameras. ${ }^{1}$ The advent of whole slide imaging (WSI) about two decades ago has provided the opportunity for pathologists to convert digitally the entire glass slides into digital or virtual slides, which gives the pathologist an opportunity to view the entire tissue section of slide in one go, which was perhaps a dream of a pathologist. ${ }^{1}$ In the last 20 years, the world has witnessed an exponential development and adoption of digital pathology (DP) techniques in various disciplines of pathology.

Integration of artificial intelligence (AI) and machine learning (ML) algorithms has further transformed the conventional norms of pathology practice, and opened a new horizon of computer-assisted diagnosis (CAD). The world observed an increasing adoption of telemedicine and telepathology. As the world grapples with the COVID-19 pandemic, increasing use of telepathology in times of lockdown, has kept the people connected and safe. Here, we briefly address the challenges of adoption of DP in developing countries including Pakistan; and discuss some solutions to overcome these challenges.

Correspondence to: Dr. Asma Shabbir, Department of Pathology, Jinnah Sindh Medical University, Karachi, Pakistan

E-mail:drasma52@gmail.com

Received: March 11, 2021; Revised: June 08, 2021;

Accepted: June 16, 2021

DOI: https://doi.org/10.29271/jcpsp.2021.09.1120
The dawn of DP has, beyond any doubt, transformed the decades-old conventional pathology practice of pathologists. The promises and results of DP are far beyond the scope of traditional microscopy. The first use of WSI was in telepathology, which allowed a pathologist to work and give consultation from any part of the world on a single click, thus reducing time and money and also the suffering of patients, who have to wait for long time for diagnosis, which is integral for their further management and treatment. This issue is more pressing in developing part of the world like Pakistan, where, there is scarcity of expert pathologists who generally prefer to live in big cities; but the bulk of population lives in scattered rural areas, where people not only suffer from malignancy and seek pathologists' opinion but also from endemic diseases; for example, tuberculosis (both pulmonary and extra-pulmonary), cutaneous leishmaniasis, malaria, typhoid etc. The role of pathologists is integral in diagnosis of these diseases. All of these endemic diseases are completely curable, but late diagnosis results in increased mortality and morbidity.

A large number of studies have validated the use of digital images for second opinion in challenging cases of surgical pathology and cytopathology with the American Telemedicine Association suggested guidelines for the adoption of telepathology. ${ }^{2,3}$ The United States' Food and Drug Administration (FDA) approved in 2017 the first use of WSI for primary diagnosis in surgical pathology. 4

The continuous and increasing requirement of physical space for storage of glass slides can also be tackled with WSI and creation of digital slides, which can store the slides for indefinite time and also can maintain their quality. WSI offers an equal prospect of standardisation of pathology education across the countries, 
particularly in developing nations, with the same slides viewed by all trainees and pathologists regardless of their geographical boundaries. WSI also permits the specialists to conduct training in interpretation of immunohistochemistry or electron microscopy and frozen section to pathology residents in distant areas having partial or no exposure to these particular fields, thus giving them equal opportunity for learning. The WSI images can be marked and connected with relevant clinical and/or radiological data, so as to offer a comprehensive view of the diagnostic approach to the clinicians. ${ }^{5}$ WSI has also removed the need of having huge class rooms with numerous light microscopes for the residents and consultants. ${ }^{5}$

Digital slides are nowadays widely used in pathology conferences and seminars to endorse collaborative learning; and offer comfort of visualisation of many images with different stains and markers in combination with clinical information. Last, but not the least, use of Al-based medical image analysis softwares have further made the work of pathologists more standardised by accurately measuring, quantifying and even picking up the small pathological lesions, which might be missed by pathologists. We did a few pilot projects by using Al software Aiforia Oy, and got appreciable number of positive results. In one of our studies, the software detected blast cells on previously diagnosed cases of chronic lymphocytic leukemia (CLL). The slides were made digital at $40 \times$ and trained the software to annotate the blast cells as green circles and red blood cells (RBCs) as red small circles (Figures 1 and 2). After giving 1000 iteration to software, it was analysed for the results. The software was able to identify the number of blast cells and RBCs in the slide.

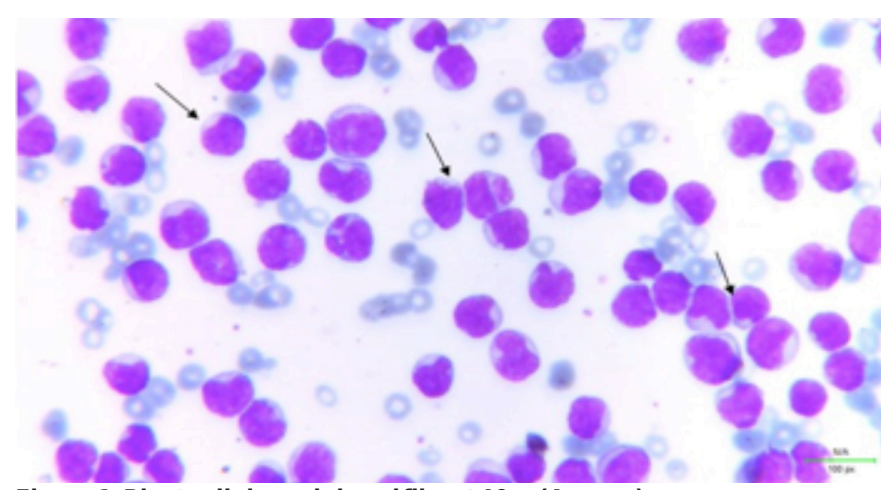

Figure 1: Blastcells in peripheral film at 40x. (Arrows).

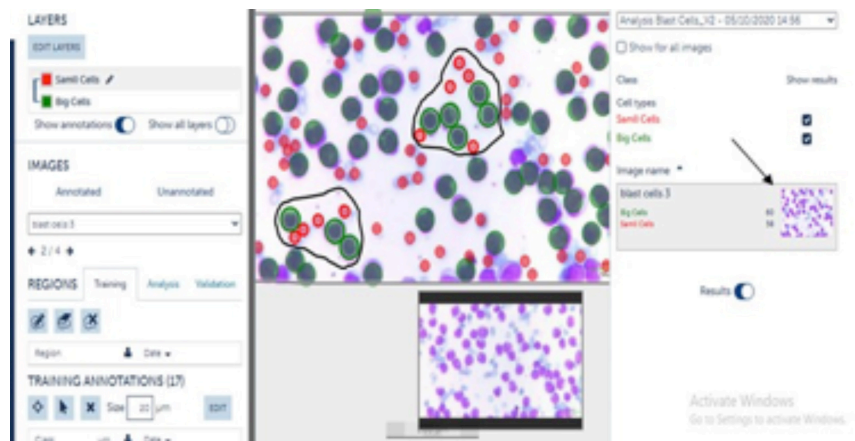

Figure 2: The software was trained to annotate blast cells as green circles and red blood cells (RBCs) as red circles. When the slide was analysed for results, it diagnosed almostall the blast cells except few of them. The software was also able to identify the number of blast cells and RBCs. (Arrow).
Despite all aforementioned advantages, still, it is prudent to recognise the several unresolved concerns related to WSI. It is also essential to know the obstacles one might face in using WSIs in clinical practice across the globe, particularly in developing countries and low resource organisations.

The primary burden of obtaining, application, and working prices of WSI scanners may be unaffordable, especially for developing nationsand low-resource settings. Additionalobligatory unseen charges of teaching of staff and pathologists, slide storage systems, and supervisory costs are also to be considered. ${ }^{6}$

A big limitation with currently available scanners is the requirement of enormous data storage capability, principally if one is viewing at 40x. Many professional companies now provide the cloud facility on economical price, which may be able to solve this issue. Beside this, to circumvent the need of scanners which are very expensive, some cheaper solutions are also available, like stitching softwares, which take a bit longer time to scan the whole slide but can be very helpful for second and third opinion, education and research purposes.

Though United States FDA has rendered its endorsement for use of WSI in surgical pathology cases, the other sub-specialties of pathology still have a long way to go to a chieve this objective.

In conclusion, WSI and Al are at our doorsteps and their adoption is now inevitable. Use of these technologies could transform the thought processes of pathologists working in developing part of the world, which contains the bulk of world disease; but is less equipped with diagnostic modalities, particularly the novel ones. Adoption of these novel methods will change the field of pathology into a highly revolutionised field, in which the role of pathologists will not be of reactionary type but they can also work on preventive health by making disease models and predict disease trends through data sciences resulting in advancement of therapeutic management of patients and personalised medicine.

\section{ETHICALAPPROVAL:}

It was a pilot study conducted on previously diagnosed cases of acute leukemia, thus the slides were taken from the archives. We carried out on a small number of slides.

\section{PATIENT'S CONSENT:}

As we did not use any of the patient's personal data, so patient consent is not required.

\section{CONFLICT OF INTEREST:}

The authors declared no conflict of interest.

\section{AUTHORS' CONTRIBUTION:}

TZ: Conceived the idea, collaborated with a software organisation, did annotation and training of cases and draft writing.

AS: Worked in draft writing, corrected the grammatical mistakes, rephrased the references and helped in analysing the results of study.

Both the authors proofread the article and approved it. 


\section{REFERENCES}

1. Wright AM, Smith D, Dhurandhar B, Fairley T, ScheiberPacht $M$, Chakraborty $S$, et al. Digital slide imaging in cervicovaginal cytology: A pilot study. Arch Pathol Lab Med 137(5):618-24, 2013. doi.org/10.5858/arpa.2012-0430-OA.

2. Rodriguez-Urrego PA, Cronin AM, Al-Ahmadie HA, Gopalan A, Tickoo SK, Reuter VE, et al. Interobserver and intraobserver reproducibility in digital and routine microscopic assessment of prostate needle biopsies. Hum Pathol 2011; 42(1):68-74. doi.org/10.1016/j.humpath. 2010.07.001.

3. Evans AJ, Krupinski EA, Weinstein RS, Pantanowitz L. 2014 American telemedicine association clinical guidelines for telepathology: Another important step in support of increased adoption of telepathology for patient care. J
Pathol Inform 2015; 6:13. doi: 10.4103/2153-3539.153906.

4. Evans AJ, Bauer TW, Bui MM, Cornish TC, Duncan H, Glassy $E F$, et al. US food and drug administration approval of whole slide imaging for primary diagnosis: A key milestone is reached and new questions are raised. Arch Pathol Lab Med 2018; 142(11):1383-7. doi: 10.5858/arpa.2017-0496CP.

5. Mubarak M. From digital to computational pathology and integrated diagnostics: The future of histopathology. J Coll Phys Surg Pak 2021; 31(1):2-3. doi: 10.29271/jcpsp.2021. 01.2 .

6. Volynskaya Z, Evans AJ, Asa SL. Clinical applications of whole-slide imaging in anatomic pathology. Adv Anat Pathol 2017; 24(4):215-21. doi: 10.1097/PAP.000000000 0000153. 